\title{
CONNECTION BETUEEN NON-ROTATING LOCAL REFERENCE FRAMES BY MEANS OF THE WORLD FUNCTION
}

\author{
J.M. GAMBI, ${ }^{1)}$ P. ROMERO, ${ }^{1)}$ A. SAN MIGUEL, ${ }^{2)}$ and F. VICENTE. ${ }^{2)}$ \\ 1) Instituto de Astronomia y Geodesia (UCM-CSIC) \\ Facultad de Ciencias Matematicas \\ Universidad Complutense, Madrid, Spain \\ 2) Departamento de Matematica Aplicada \\ Universidad de Valladolid, Valladolid, Spain
}

ABSTRACT. By means of the world function an approximate transformation showing the Riemann tensor between the Fermi coordinates associated to two non-rotating local reference frames is derived in a General Relativistic space-time. One of the observer's world lines is resticted to be a time-like geodesic of the space-time, and the other is a timelike curve of a general character. The space-time where the transformation is evaluated is supposed to be of small curvature, and the calculations are carried out in a first order of approximation with respect to the Riemann tensor.

\section{Introduction}

The technique based on the world function can be found in Synge (1960). The transformation is derived in three steps: first, under the restrictions of this technique a general transformation is obtained. Next, the small-curvature hypothesis on the space-time is applied; and finally, the hypothesis of quasi-parallelism between the base lines of the reference frames is applied to derive the result.

\section{General Transformation}

Denoting by $\Pi$ a time-like curve and by $\Gamma$ a time-like geodesic, both in a region $\mathcal{N}$ where the cited technique can be used; parametrizing these base lines with the afin parameters $s$ and $\mathbf{s}^{\prime}$, so that $A$ and $D$ represent two generic points in $\Pi$ and $\Gamma$ respectively; denoting by $n^{1} A$ and $n^{1} D$ the unit tangent vectors of $\Pi$ and $\Gamma$ at $A$ and $D$ respectively; and finally, denoting by $\Sigma(A)$ and $\Sigma(D)$ the hypersurfaces of $N$ defined by

$$
\Sigma(\mathrm{A}):=\left\{\mathrm{P} \in \mathcal{N} / \mathrm{n}_{1_{\mathrm{A}}} \Omega^{1} \mathrm{~A}(\mathrm{AP})=0\right\}, \quad \Sigma(\mathrm{D}):=\left\{\mathrm{Q} \in \mathcal{N} / \mathrm{n}_{\mathrm{I}_{\mathrm{D}}} \Omega^{1} \mathrm{D}(\mathrm{DQ})=0\right\}
$$


then the Fermi coordinates of a point $B \in \Sigma(A) \cap \Sigma(D)$ can be written in terms of the world function, $\Omega$, in the following way : First, with respect to the base 1 ine $\Pi$ and to the tetrad $\lambda_{(a)}^{1}$ Fermi transported along $\Pi$, which we shall denote by $x^{(\alpha)}(B)=x_{(\alpha)}(B), x^{(4)}(B)=-x_{(4)}(B)=s$, as

$$
\left.\mathrm{x}^{(\alpha)}(\mathrm{B})\right|_{\mathrm{A}}=\left.\mathrm{X}_{(\alpha)}(\mathrm{B})\right|_{\mathrm{A}}=-\Omega_{\mathrm{A}_{A}}(\mathrm{AB}) \lambda_{(\alpha)}^{{ }_{\mathrm{A}}},\left.\mathrm{X}^{(4)}(\mathrm{B})\right|_{\mathrm{A}}=-\left.\mathrm{x}_{(4)}(\mathrm{B})\right|_{\mathrm{A}}=\mathrm{s}
$$

and second, with respect to the geodesic line $\Gamma$ and the parallel transported tetrad $\lambda_{(a)}^{\prime 1}$ along $\Gamma$, which will be denoted by $x^{,(\alpha)}(B)=$ $x^{\prime}(\alpha)(B), x^{\prime(4)}(B)=-x_{(4)}^{\prime}(B)=s^{\prime}$, as

$$
\left.X^{(\alpha)}(B)\right|_{D}=\left.X_{(\alpha)}(B)\right|_{D}=-\Omega_{1_{D}}(D B) \lambda_{(\alpha)}^{1},\left.\quad X^{(4)}(B)\right|_{D}=-\left.X_{(4)}(B)\right|_{D}=s^{\prime}
$$

where $\Omega_{1}$ and $\Omega_{1}$ are the covariant derivatives of $\Omega$ with respect to $A$ and $D$ respectively.

In order to obtain the relationship between the Fermi coordinates $\left[x_{(\alpha)}(B), x_{(4)}(B)\right]$ and $\left[x_{(\alpha)}^{\prime}(B), x_{(4)}^{\prime}(B)\right]$ we consider the point $C$, intersection of $\Sigma(A)$ and $\Gamma$, and it is supposed that $\left.X_{(4)}(C)\right|_{D}=-\Delta s^{\prime}$. Then, by using the two solutions for the geodesic triangles ADB and ACD, and the parallel propagator, $g_{1_{A} J_{D}}$, applied to the tetrad $\lambda_{(a)}^{1}$, we have

$$
\begin{aligned}
& \left.X_{(\alpha)}(B)\right|_{A}-\left.X_{(\alpha)}(C)\right|_{A}-X_{(\beta)}(B) I_{D} L_{(\alpha)}^{(\beta)}=\Omega_{I_{D}}(C D) L_{(\alpha)}^{(4)} \lambda_{(4)}^{1} \\
& -\left[\chi_{1_{A}}(\mathrm{ABD})-\chi_{1_{A}}(\mathrm{ACD})\right] \lambda_{(\alpha)}^{{ }^{1}}+\left[\chi_{1_{D}}(\mathrm{ABD})-\chi_{1_{D}}(\mathrm{ACD})\right] L_{(\alpha)}^{(b)} \lambda_{(b)}^{1} \\
& +\frac{1}{2}\left[\phi_{1_{A}}(A B D)-\phi_{1_{A}}(A C D)\right] \lambda_{(\alpha)}^{1}-\frac{1}{2}\left[\phi_{1}(A B D)-\phi_{1_{D}}(A C D)\right] L_{(\alpha)}^{(b)} \lambda_{(b)}^{1}, D
\end{aligned}
$$

where $\chi(A B D), \chi(A C D), \phi(A B D)$ and $\phi(A C D)$ are the 3-point invariants given by

$$
\begin{gathered}
\chi(A B D)=\Omega_{1_{B}}(A B) \Omega^{1}{ }^{B}(B D), \quad \chi(A C D)=\Omega_{1_{C}}(A C) \Omega^{1}{ }^{1}(C D), \\
\phi(A B D)=\frac{1}{3} \int_{0}^{1}(1-\zeta)^{3} \frac{d^{4} \Omega(\zeta)}{d \zeta^{4}} d \zeta, \quad \phi(A C D)=\frac{1}{3} \int_{0}^{1}(1-\theta)^{3} \frac{d^{4} \Omega(\theta)}{d \theta^{4}} d \theta
\end{gathered}
$$




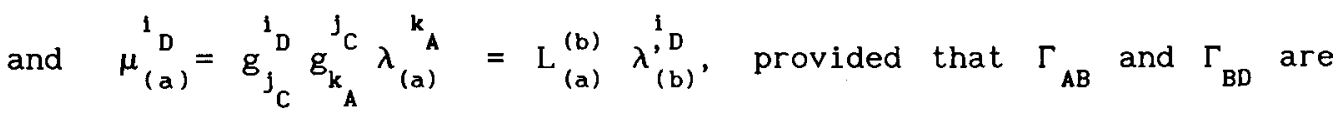
parametrized so that $\zeta$ takes the values $O$ at $B$, and 1 both at $A$ and $D$, and that $\Gamma_{\mathrm{CA}}$ and $\Gamma_{\mathrm{CD}}$ are parametrized so that $\theta=0$ at $C$, and $\theta=1$ at $A$ and $D$.

\section{Approximation by small curvature}

By introducing the hypothesis that at any point $P$ of the hypersurfaces $\Sigma(A)$ and $\Sigma(D)$ the Riemann tensor, $R_{1 j k 1}$, and its covariant derivatives are small, of the first order, or $\mathrm{O}_{1}$, and denoting by $\mathrm{O}_{2}$ second-order smalles with respect to the Riemann tensor, we obtain

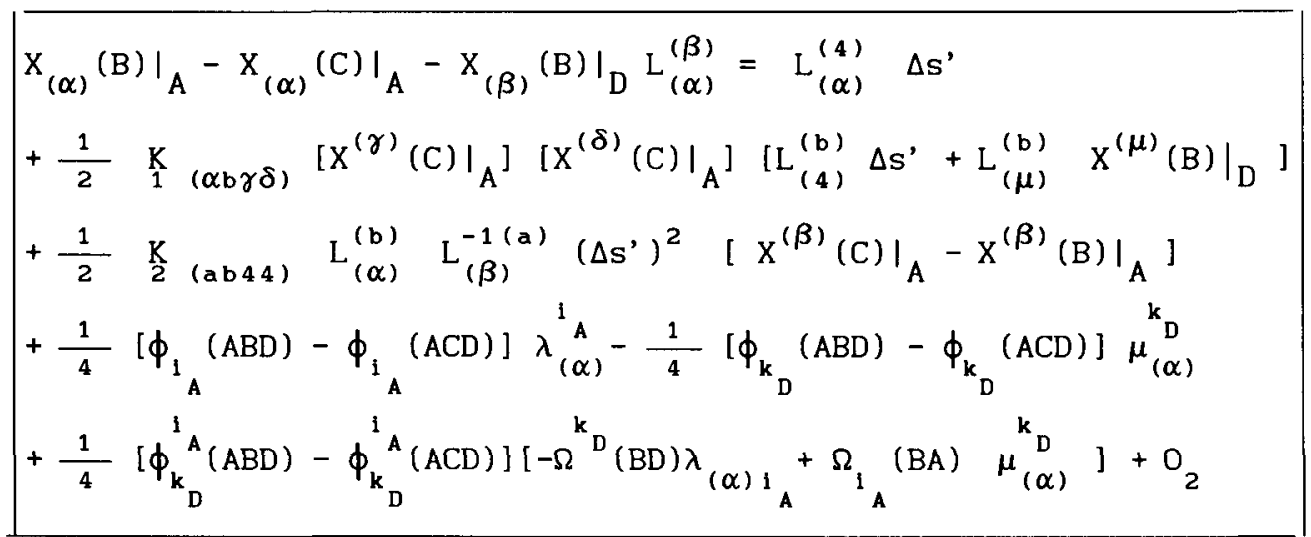

where

$$
\mathrm{K}_{(\mathrm{ab \gamma} \delta)}=-\frac{3}{2} \int_{0}^{1}\left(1-\sigma_{1}\right) \sigma_{1} \mathrm{~S}_{(\mathrm{ab \gamma} \delta)} \mathrm{d} \sigma_{1}, \quad \mathrm{~K}_{2}\left(\mathrm{ab44)}=-\frac{3}{2} \int_{0}^{1}\left(1-\sigma_{2}\right) \sigma_{2} S_{(a b 44)} \mathrm{d} \sigma_{2}\right.
$$

the first integral being taken along the geodesic $\Gamma_{A C}$, the second along the geodesic $\Gamma_{C D}\left(S_{(a b c d)}\right.$ being the symmetrized Riemann tensor) and

$$
\phi(\mathrm{ABD})=\phi_{0}(\mathrm{ABD})+\phi_{1}(\mathrm{ABD})+\phi_{2}(\mathrm{ABD})+\mathrm{O}_{2}
$$

$\phi_{0}(A B D)=3 k^{3} \int_{0}^{1}(1-\zeta)^{3} d \zeta \int_{u_{1}}^{u_{2}}\left[\left(u_{2}-u\right)^{2}+\left(u-u_{1}\right)^{2}\right]\{1122\} d u$

$\phi_{1}(A B D)=2 k^{3} \int_{0}^{1} \zeta(1-\zeta)^{3} d \zeta \int_{u_{1}}^{u} 2\left[2\left(u_{2}-u\right)^{3}\{11221\}+3\left(u_{2}-u\right)^{2}\left(u-u_{1}\right)\{11222\}\right.$

$\left.+3\left(u_{2}-u\right)\left(u-u_{1}\right)^{2}\{22111\}+2\left(u-u_{1}\right)^{3}\{22112\}\right] d u$ 


$$
\begin{aligned}
\phi_{2}(A B D) & =\frac{1}{2} k^{3} \int_{0}^{1} \zeta^{2}(1-\zeta)^{3} d \zeta \int_{u_{1}}^{u}\left[\left(u_{2}-u\right)^{4}\{112211\}+4\left(u_{2}-u\right)^{3}\left(u-u_{1}\right)\{112212\}\right. \\
& +3\left(u_{2}-u\right)^{2}\left(u-u_{1}\right)^{2}(\{112222\}+\{221111\}) \\
& \left.+4\left(u_{2}-u\right)\left(u-u_{1}\right)^{3}\{221121\}+\left(u_{-}-u_{1}\right)^{4}\{221122\}\right] d u, \quad k^{-1}=u_{2}-u_{1}
\end{aligned}
$$

with a similar expression for $\phi(A C D)$, where

$\{1122 \ldots\}=S_{(a b c d)}\left[\left.X^{(a)}(C)\right|_{A}\right]\left[\left.X^{(b)}(C)\right|_{A}\right]\left[\left.X^{(c)}(D)\right|_{A}\right]\left[\left.X^{(d)}(D)\right|_{A}\right][\ldots]$

\section{Approximation by quasiparallelism}

Denoting by $U^{1} \mathrm{C}$ the tangent vector to the geodesic $\Gamma$ at $C$, by $U^{{ }^{A}}$ the tangent vector to $\Pi$ at $A$, and introducing the hypothesis of quasi-parallelism, i.e.,

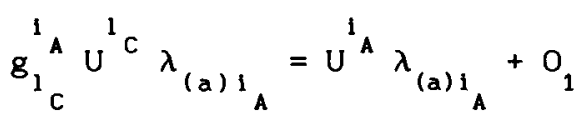

then the final transformation reads

$$
\begin{aligned}
& x_{(\alpha)}(B)-x_{(\alpha)}(C)-x^{\prime}{ }_{(\beta)}(B) L_{(\alpha)}^{(\beta)}=\left[1_{(\alpha)}^{(4)}+\bar{K}_{1}{ }_{(\alpha 4)}\right] \Delta s^{\prime} \\
& +\overline{\mathrm{K}}_{(\alpha \beta)} \mathrm{L}_{(\mu)}^{(\beta)} \mathrm{x}^{,(\mu)}(\mathrm{B})+\frac{1}{4}\left[\phi_{1}(\mathrm{ABD}) \lambda_{(\mathrm{A})}{ }^{\mathrm{A}}-\phi_{\mathrm{k}_{\mathrm{D}}}(\mathrm{ABD}) \mathrm{L}_{(\alpha)}^{(\beta)} \lambda_{(\beta)}{ }^{\mathrm{k} D}\right] \\
& +\frac{1}{4}\left[\phi_{k_{D}}^{1}(A B D)\right]\left[x_{(\beta)}^{\prime}(B) \lambda^{k^{\prime} D(\beta)} \lambda_{(\alpha) i_{A}}-x_{(\gamma)}(B) \lambda_{1_{A}}^{(\gamma)} L_{(\alpha)}^{(\beta)} \lambda_{(\beta)}^{\lambda^{, D}}\right] \\
& +\mathrm{O}_{2}
\end{aligned}
$$

where

$$
{\underset{1}{\mathrm{~K}}}_{\left(\alpha_{4}\right)}=\frac{1}{2}{\underset{1}{\mathrm{~K}}\left(\alpha_{4} \gamma \delta\right)} \mathrm{x}^{(\gamma)}(\mathrm{C}) \mathrm{x}^{(\delta)}(\mathrm{C}), \overline{\mathrm{K}}_{1(\alpha \beta)}=\frac{1}{2} \mathrm{~K}_{1}^{\mathrm{K}}(\alpha \beta \gamma \delta)^{(\gamma)}(\mathrm{C}) \mathrm{x}^{(\delta)}(\mathrm{C})
$$

and $\mathrm{L}=1+\mathrm{O}_{2}$.

This transformation is ready to input the value of the Riemann tensor evaluated on the accelerated observer and the behaviour of the two reference frames as characteristics derived from their particular selection and from the dynamical model to be chosen.

\section{References}

Synge, J. L. 1960, Relativity: The General Theory, North Holland, Amsterdam 